\title{
A reversibly photoswitchable GFP-like protein with fluorescence excitation decoupled from switching
}

\author{
Tanja Brakemann ${ }^{1,6}$, Andre C Stiel $^{1,6}$, Gert Weber $^{2}$, Martin Andresen $^{1}$, Ilaria Testa ${ }^{1}$, Tim Grotjohann $^{1}$, \\ Marcel Leutenegger ${ }^{1}$, Uwe Plessmann ${ }^{3}$, Henning Urlaub ${ }^{3,4}$, Christian Eggeling ${ }^{1}$, Markus C Wahl' ${ }^{2}$, \\ Stefan W Hell ${ }^{1} \&$ Stefan Jakobs ${ }^{1,5}$
}

\begin{abstract}
Photoswitchable fluorescent proteins have enabled new approaches for imaging cells, but their utility has been limited either because they cannot be switched repeatedly or because the wavelengths for switching and fluorescence imaging are strictly coupled. We report a bright, monomeric, reversibly photoswitchable variant of GFP, Dreiklang, whose fluorescence excitation spectrum is decoupled from that for optical switching. Reversible on-and-off switching in living cells is accomplished at illumination wavelengths of $\sim 365 \mathrm{~nm}$ and $\sim 405 \mathrm{~nm}$, respectively, whereas fluorescence is elicited at $\sim 515 \mathrm{~nm}$. Mass spectrometry and highresolution crystallographic analysis of the same protein crystal in the photoswitched on- and off-states demonstrate that switching is based on a reversible hydration/dehydration reaction that modifies the chromophore. The switching properties of Dreiklang enable far-field fluorescence nanoscopy in living mammalian cells using both a coordinate-targeted and a stochastic single molecule switching approach.
\end{abstract}

Fluorescent proteins (FPs) ${ }^{1}$ whose fluorescence can be reversibly or irreversibly switched by optical irradiation have opened new opportunities for the imaging of cells. They have facilitated in vivo protein-tracking schemes ${ }^{2,3}$, applications based on singlemolecule observations $s^{4,5}$ and fluorescence microscopy with subdiffraction resolution ${ }^{6-10}$.

Still, photoswitchable proteins have not displayed their full potential, because proteins that are just photoactivatable ${ }^{11-13}$ can be switched only once, which implies that repeated measurements with the same molecule are impossible. On the other hand, photochromic or reversibly switchable fluorescent proteins (RSFPs) can be repeatedly photoswitched between a fluorescent and a nonfluorescent state by irradiation with light of two different wavelengths. However, in all previously characterized RSFPs, the wavelength used for generating the fluorescence emission is identical to one of the wavelengths used for switching the fluorescence on or off. The result is a complex interlocking of switching and fluorescence readout ${ }^{14-22}$, impeding or even precluding many applications, including fluorescence nanoscopy (super-resolution microscopy). Hence, the identification of an RSFP in which the generation of fluorescence is disentangled from switching has long been pursued.

\section{RESULTS}

\section{Generation of the RSFP Dreiklang}

Numerous GFP variants exhibit some degree of (generally undesirable) reversible photoswitching $4,23,24$. We found that the fluorescence of the yellow fluorescent protein Citrine ${ }^{25,26}$, a derivative of GFP, can be reversibly modulated to a small extent by alternate irradiation with light of $365 \mathrm{~nm}$ (on switching) and $405 \mathrm{~nm}$ (off switching), whereas fluorescence is excited at $515 \mathrm{~nm}$. However, the achievable contrast was low, especially at $\mathrm{pH}$ values $>6$, rendering the reversible switching of Citrine unusable (Supplementary Fig. 1).

To further develop this unusual switching behavior, we performed extensive random mutagenesis as well as directed PCR-mediated mutagenesis on a plasmid encoding Citrine. We transformed Escherichia coli with the plasmid, and screened with an automated home-built fluorescence microscope for bacterial colonies expressing fluorescent proteins whose fluorescence was excited with green light $(515 \mathrm{~nm})$ and which could be reversibly photoswitched from a fluorescent state to a long-lived nonfluorescent state by irradiation with near-UV (405 nm) light and back to a fluorescent state by UV (365 nm) light (Fig. 1a). In several consecutive screening rounds $\sim 70,000$ individual clones were analyzed. Finally, we identified a mutant differing from Citrine at four positions (Citrine-V61L, F64I, Y145H, N146D) (Supplementary Fig. 2), which can be effectively switched and excited to fluoresce. We named this switchable fluorescent protein Dreiklang, the German word for a three-note chord in music.

At thermal equilibrium, Dreiklang adopts the brightly fluorescent on-state, with a quantum yield of 0.41 and an extinction coefficient of $83,000 \mathrm{M}^{-1} \mathrm{~cm}^{-1}$ at $\mathrm{pH} 7.5$ (Table 1). In the on-state, it exhibits two absorption bands (peaking at $412 \mathrm{~nm}$ and $511 \mathrm{~nm}$ ), corresponding to the neutral (protonated) and ionized (deprotonated) states of the chromophore, respectively (Fig. $\mathbf{1 b})^{1}$. The $\mathrm{p} K_{a}$ of the on-state chromophore is 7.2 , which is $1.5 \mathrm{pH}$ units higher than the $\mathrm{p} K_{a}$ of

\footnotetext{
${ }^{1}$ Max Planck Institute for Biophysical Chemistry, Department of NanoBiophotonics, Göttingen, Germany. ²Freie Universität Berlin, Institut für Chemie und Biochemie, AG Strukturbiochemie, Berlin, Germany. ${ }^{3}$ Max Planck Institute for Biophysical Chemistry, Bioanalytical Mass Spectrometry, Göttingen, Germany. ${ }^{4}$ University Medical Center Göttingen, Department of Clinical Chemistry, Bioanalytics, Göttingen, Germany. ${ }^{5}$ University of Göttingen Medical School, Göttingen, Germany. ${ }^{6}$ These authors contributed equally to this work. Correspondence should be addressed to S.W.H. (shell@gwdg.de) or S.J. (sjakobs@gwdg.de).
} 
Figure 1 Properties of Dreiklang. (a) Scheme depicting Dreiklang's switching modality. (b) Normalized absorbance (solid black line), fluorescence emission (dashed green line) and fluorescence excitation (dotted red line) spectra of the (fluorescent) equilibrium-state Dreiklang at $\mathrm{pH}$ 7.5. (c,d) Switching curves of Dreiklang's fluorescence recorded on colonies of living E. coli. Off- and on-switching was performed with near-UV (405 nm) and UV light (365 nm), respectively, and fluorescence read-out with green light $(515 \mathrm{~nm})$. The respective irradiation scheme is indicated on top of the graphs by the colored bars. (c) One switching cycle. Fluorescence was continuously recorded. (d) Twenty consecutive switching cycles. Fluorescence was recorded when the cells were irradiated with green light only. (e) Irradiationdependent changes in Dreiklang absorbance. Absorbance spectra obtained at the indicated time points during switching of equilibriumstate Dreiklang $(\mathrm{pH} 7.5)$ into the off-state by irradiation with $405 \mathrm{~nm}$. (f) Irradiationindependent changes in Dreiklang fluorescence due to the thermal equilibration from the offstate into the fluorescent equilibrium state. After off-switching, fluorescence was recorded at $25{ }^{\circ} \mathrm{C}$ under constant irradiation with $515 \mathrm{~nm}$ (black line) or by consecutive 20 -ms pulses of $515 \mathrm{~nm}$ light in $60 \mathrm{~s}$ intervals (red dots). The similar curves demonstrate that $515 \mathrm{~nm}$ light does not photoswitch Dreiklang.

Inset: relaxation half-time from the off- into

the equilibrium-state as a function of temperature. The data were obtained on purified Dreiklang ( $\mathrm{pH}$ 7.5) (circles) or on living cells expressing Dreiklang targeted to the ER (squares). Red line: single exponential fit to the data obtained on purified protein. a
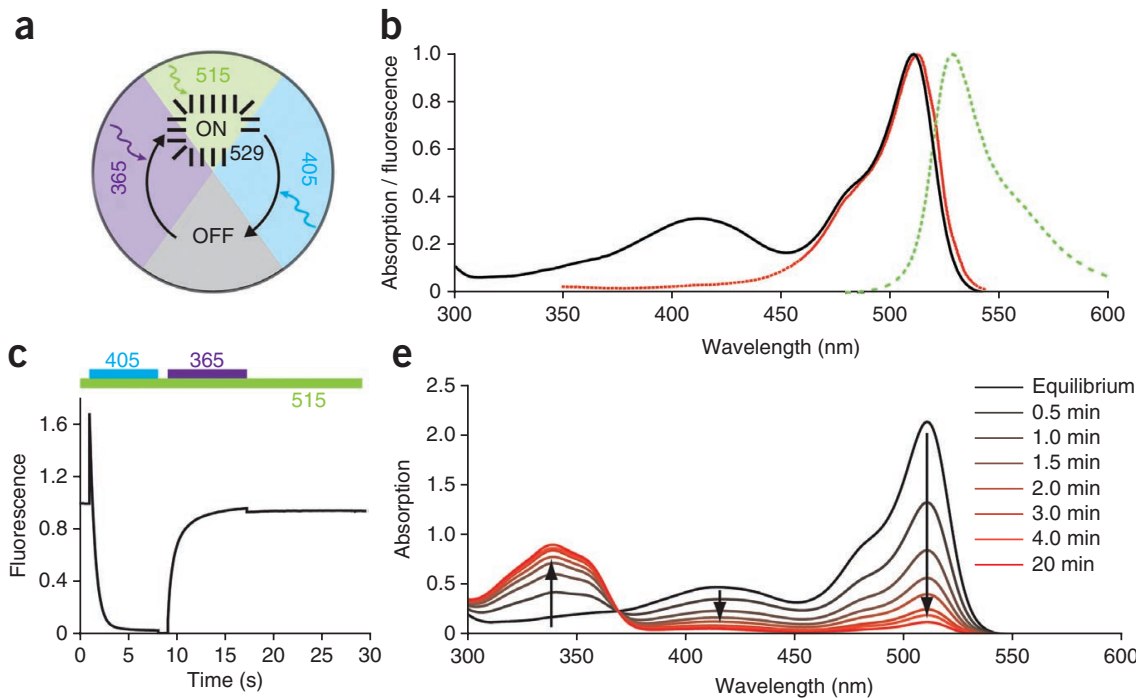

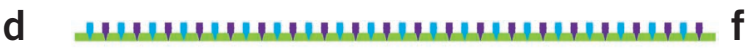

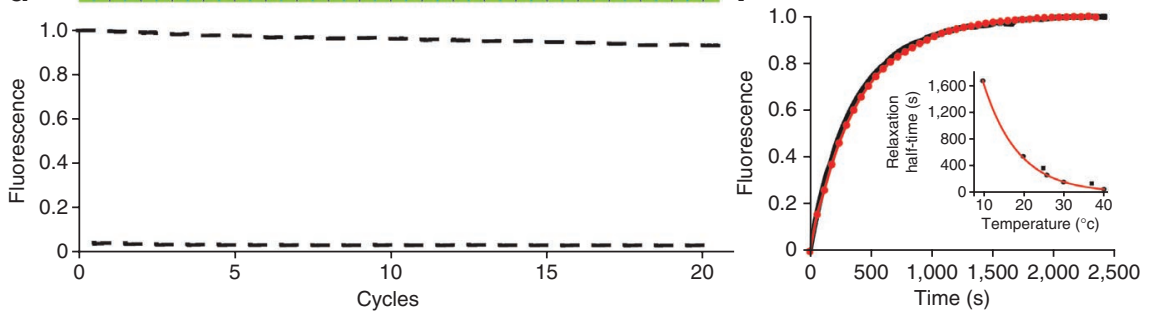

Citrine (5.7) (Supplementary Fig. 1c,d) ${ }^{26}$. Hence, an important role of the four mutations that discriminate Dreiklang from Citrine is to shift the $\mathrm{p} K_{a}$ of the on-state chromophore.

Excitation with $500 \mathrm{~nm}$ light results in fluorescence with an emission maximum at $529 \mathrm{~nm}$ (Fig. 1b). Irradiation at the absorption band of the neutral state with light of $405 \mathrm{~nm}$, switched the protein to a nonfluorescent off-state (Fig. 1c,d). Upon photoswitching to the off-state, a new and unusual absorption band at $340 \mathrm{~nm}$ appeared, whereas the absorption bands corresponding to the on-state disappeared (Fig. 1e). Subsequent illumination at the $340-\mathrm{nm}$ absorption band of the offstate switched the protein back into the on-state (Fig. 1c,d). Switching Dreiklang on at $365 \mathrm{~nm}$ and off at $405 \mathrm{~nm}$ was reversible (Fig. 1d). As expected from the $\mathrm{p} K_{a}$ of the on-state chromophore, the fluorescence of Dreiklang could be reversibly switched with a good contrast in a pH range from 6 to 9 (Supplementary Fig. 1).

Effective switching could be performed at all physiological temperatures applied $\left(10-40{ }^{\circ} \mathrm{C}\right)$ and was accelerated with increasing temperatures, although the maximal fluorescence was concomitantly reduced at higher temperatures (Supplementary Fig. 3). Likewise, the thermal relaxation from the optically induced nonfluorescent state into the equilibrium on-state depends on temperature (Fig. 1f, inset).

Table 1 Properties of Citrine and Dreiklang

\begin{tabular}{lcccrc}
\hline & $\begin{array}{c}\text { Absorbance } \\
(\mathrm{nm})\end{array}$ & $\begin{array}{c}\text { Emission } \\
(\mathrm{nm})\end{array}$ & QY & $\begin{array}{c}\varepsilon-\mathrm{max} \\
\left(\mathrm{M}^{-1} \mathrm{~cm}^{-1}\right)\end{array}$ & Equilibrium \\
\hline Citrine & 515 & 533 & 0.54 & 132,000 & On \\
Dreiklang & $340^{\text {off }} / 412^{\text {on }} / 511^{\text {on }}$ & 529 & 0.41 & 83,000 & On \\
\hline
\end{tabular}

Quantum yield $(Q Y)$ and extinction coefficient $(\varepsilon)$ were measured for the fluorescent on-state protein at $\mathrm{pH} 7.5$.
Thermal equilibration was not affected by irradiation at $515 \mathrm{~nm}$ $\left(0.82 \mathrm{~W} / \mathrm{cm}^{2}\right)$, thus, exciting fluorescence, affected neither the nonfluorescent nor the fluorescent state (Fig. 1f), although noticeable cross-talk to the absorption band of the neutral state is anticipated at higher intensities. In living E. coli cells expressing Dreiklang, we recorded $\sim 160$ switching cycles before the fluorescence was reduced to $50 \%$ (at $1.4 \mathrm{~W} / \mathrm{cm}^{2}, 365 \mathrm{~nm} ; 110 \mathrm{~W} / \mathrm{cm}^{2}, 405 \mathrm{~nm} ; 0.82 \mathrm{~W} / \mathrm{cm}^{2}, 515 \mathrm{~nm}$ ) (Supplementary Fig. 4), demonstrating its high switching endurance.

The on-off contrast of the fluorescence signal depends on the sample and the light intensities applied. In widefield images of living mammalian cells, the off-state background signal was $<1.4 \%$ of the on-state signal (see below), whereas in thick layers of E. coli cells, which were used for screening, it was generally 5-10\% (Fig. 1d and Supplementary Fig. 4). In the latter case, we attribute the background mainly to scattering, rather than to ineffective switching.

On native gels, Dreiklang behaves as a monomer (Supplementary Fig. 5). Its maturation half-time is $\sim 1.2 \mathrm{~h}$ at $30{ }^{\circ} \mathrm{C}$ and $\sim 2 \mathrm{~h}$ at $37^{\circ} \mathrm{C}$ (Supplementary Fig. 6). At $\mathrm{pH}=7.5$, Dreiklang is more resistant to photobleaching than its parent Citrine when using the same light intensities for exciting fluorescence, which may be partly due to Dreiklang's lower extinction coefficient (Supplementary Fig. 7).

\section{Light-driven switching of Dreiklang}

To determine the molecular basis for the reversible switching of Dreiklang, we solved the structures of the light-induced off-state $(1.7 \AA)$ and on-state $(2.0 \AA)$ using the same protein crystal. To this end, a Dreiklang protein crystal in a buffer of $\mathrm{pH} 4.6$ was switched at room temperature $(295 \mathrm{~K})$ from the fluorescent equilibrium state into the offstate by irradiation at $405 \mathrm{~nm}$ until fluorescence reached a minimum. 
Figure 2 Molecular basis of Dreiklang photoswitching. (a) Dreiklang in the fluorescent equilibrium-state (top), the nonfluorescent off-state (middle) and the fluorescent on-state (bottom). Left, top: representative Dreiklang protein crystal. Left, bottom: proposed chemical structure of the chromophore. Central: details of the X-ray structures (PDB IDs: 3ST2, 3ST3, 3ST4, respectively, top to bottom). Shown is the chromophore (carbon, magenta/gray; oxygen, red; nitrogen, blue). In the equilibrium-state and the on-state, water Wat $_{a}$ (magenta sphere) is additionally displayed. Final $2 F_{0}-F_{\mathrm{c}}$ electron densities are contoured at the $1 \sigma$ level. The off-state and the on-state structures have been successively recorded on the same protein crystal. Right: representative deconvoluted ESI-MS spectra of Dreiklang photoswitched in solution and measured under native conditions. (b) Overall Dreiklang ribbon structure displayed in two orthogonal views. (c) Chromophore and immediate surrounding of on-state Dreiklang (magenta) and GFP (PDB: 1EMA ${ }^{25}$ ) (cyan). The Van-der-Waals' radii of important atoms are indicated by spheres to highlight structural restraints. The chromophores are depicted as ball and stick whereas the surrounding amino acid residues are shown in the stick representation. (d) Superimposed representations of the Dreiklang hydrogen bond network in the (fluorescent) equilibrium- and the off-states. Equilibrium-state carbons, magenta; off-state carbons, gray; oxygen, red; nitrogen, blue. Important water molecules are shown as magenta (equilibrium-state) and gray (off-state) spheres. Inset: hydrogen bond network in GFP. a
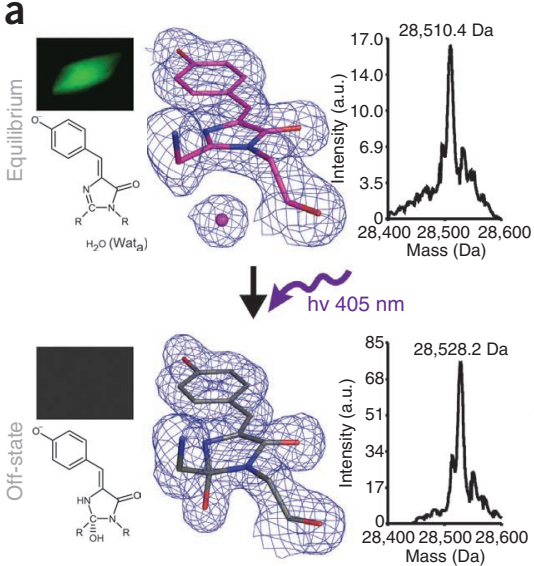

$\downarrow \sim \sim \sim m$ hv $365 \mathrm{~nm}$
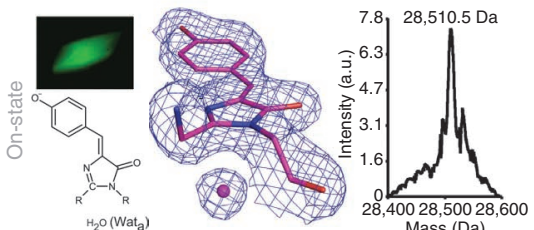

C
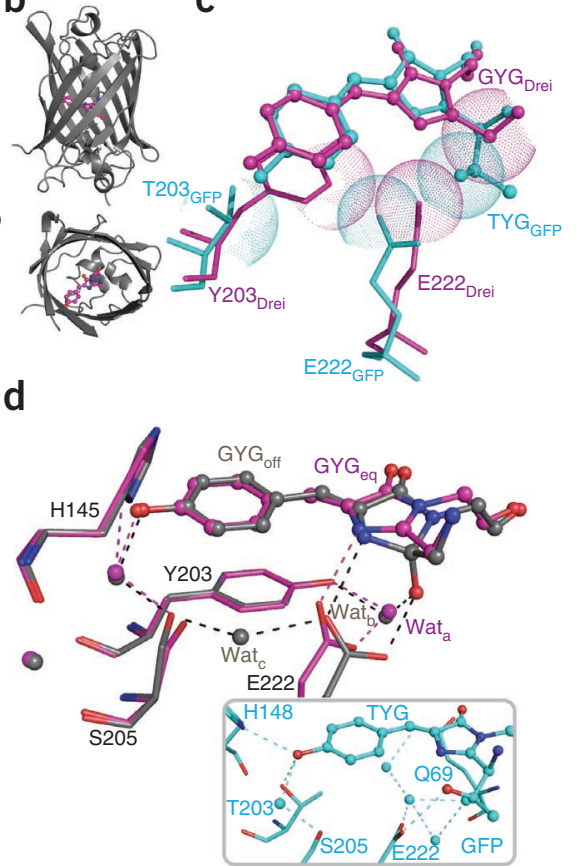

After the off-state diffraction data was recorded at $100 \mathrm{~K}$, we warmed the very same crystal of Dreiklang back to $295 \mathrm{~K}$, switched it by irradiation with $365 \mathrm{~nm}$ light until the fluorescence reached a maximum and recorded the on-state diffraction data at $100 \mathrm{~K}$. In addition, we solved the X-ray structure of Dreiklang in the fluorescent equilibrium-state to a resolution of $1.9 \AA$ (Fig. 2). Notably, the kinetics of the thermal equilibration of the fluorescence signal after switching off was comparable for Dreiklang in solution and in the crystal (Supplementary Fig. 8), indicating that the crystal lattice did not have major effects on the switching behavior.

The overall structure of Dreiklang resembles that of GFP and related proteins (Fig. 2b). The chromophore, autocatalytically formed from the Gly65-Tyr66-Gly67 tripeptide, resides in an alpha-helical segment, enclosed by an 11-stranded beta-barrel. As expected from the similar spectroscopic properties, the on-state structure was practically superimposable on the fluorescent equilibrium-state structure. The on-state chromophore consists of an imidazolinone-ring, connected by a methine bridge to a $p$-hydroxyphenyl ring. The two rings of the chromophoric systems were largely co-planar, facilitating a conjugated pi-electron system and hence supporting fluorescence.

In the off-state, the $p$-hydroxyphenyl ring lies largely in plane with the $\mathrm{C}_{\alpha 66} \mathrm{C}_{\beta 66}$ bond, as well as with the $\mathrm{C}_{\alpha 66}-\mathrm{C}_{66}$ and the $\mathrm{C}_{\alpha 66}-\mathrm{N}_{66}$ bonds, indicating that the methine bridge connecting the two rings is maintained. However, in the off-state structure, the planarity of the five-membered ring was markedly distorted with the chromophoric $\mathrm{C}_{65}$ exhibiting a tetrahedral geometry indicative of an $\mathrm{sp}^{3}$ hybridization. A clear signal in the electron density map indicates a new hydroxyl group at the $\mathrm{C}_{65}$ atom, suggesting that the imidazolinone ring was converted into a 2-hydroxyimidazolidinone ring (Fig. 2a and Supplementary Fig. 9). We propose that the hydration of the imidazolinone ring shortens the chromophoric pi-electron system, resulting in the new absorption band at $340 \mathrm{~nm}$ and the simultaneous disappearance of the absorption bands at 412 and $511 \mathrm{~nm}$ (Fig. 1e).
To further confirm this light-induced chemical modification, we carried out electrospray ionization mass spectrometry (ESI-MS). To this end, switching of Dreiklang in solution ( $\mathrm{pH} \mathrm{6.9;295} \mathrm{K)} \mathrm{was}$ monitored by measuring the fluorescence signal; the proteins in the respective states were immediately analyzed by ESI-MS under native conditions (in 18\% acetonitrile). We found a mass difference of $18 \pm$ $0.3 \mathrm{Da}$ between the nonfluorescent state and the light-induced onstate or the equilibrium state, respectively (Fig. 2a and Supplementary Figs. 10 and 11). This strongly indicates the reversible covalent addition of a water molecule that occurred parallel to changes in the fluorescence signal. Hence, the ESI-MS data are in full agreement with the $\mathrm{X}$-ray data, supporting the view of a reversible light-induced covalent chemical modification, that is, a hydration-dehydration reaction of the chromophoric five-membered ring as the underlying molecular mechanism of switching in Dreiklang.

A similar reversible hydration reaction was postulated, although controversially discussed, to occur during the chromophore formation of GFP ${ }^{27-29}$. This might suggest that the light-induced reversible switching of Dreiklang is based on a molecular reaction that is possibly occurring during chromophore maturation of some fluorescent proteins. Hence, we propose that Dreiklang may be used as a scaffold for further engineering and that this switching mechanism may be transferred to other fluorescent proteins.

Our mutagenesis studies showed that the amino acid residues Y203 and E222 as well as the chromophore building G65 are crucial for the unusual switching characteristics of Dreiklang. The amino acids G65 and Y203 facilitate the positioning of the side chain of E222 close to the imidazolinone ring (Fig. 2c). In the fluorescent-state, Y203 and E222 form hydrogen bonds to a water molecule ( $\mathrm{Wat}_{\mathrm{a}}$ ) and thereby stabilize it in close vicinity to the $\mathrm{C}_{65}$ of the chromophore (Fig. 2d), a situation that is different in the nonswitchable GFP (avGFP-S65T) ${ }^{25}$ (Fig. 2d, inset). In GFP, a water molecule corresponding to $\mathrm{Wat}_{\mathrm{a}}$ is stabilized by water-mediated $\mathrm{H}$-bonds only. We propose that $\mathrm{Wat}_{\mathrm{a}}$ is 
Figure 3 Applications of Dreiklang. (a) 'PacMan' movie. Thirty-three individual images were written successively at the same position of a polyacrylamide-Dreiklang layer. Before writing each new frame, all molecules were photoswitched to the on-state. Shown are the first and the thirty-third frame (see also Supplementary Movie 1). Scale bar, $100 \mu \mathrm{m}$. (b) Switching of various Dreiklang fusion proteins in living Vero cells (from left to right, on, off, etc.). From top to bottom: DreiklangMAP2, Dreiklang- $\alpha$-tubulin, mito-Dreiklang, Dreiklang-Histone2B. Fluorescence was excited with green light (495 nm); switch-off (light blue arrowheads): near-UV (420 nm); switch-on (violet arrowheads): UV-light $(360 \mathrm{~nm})$.

(c) FRAS with Dreiklang. Dreiklang was targeted to the ER in living Vero cells. Images from top to bottom: overview; before switching; immediately after switching Dreiklang off in

region of interest (ROI)-i; and at the end of the measurement ( $65 \mathrm{~s}$ after switching). Graphs on the right: the plotted fluorescence signals were collected within the indicated ROIs during 20 repetitions of the same FRAS experiment on a single cell (ROI-i: in this region Dreiklang was selectively switched off prior to the measurement; ROI-ii: in the same cell, showing the flow of switched-off Dreiklang molecules into this region; ROI-iii: in the neighboring cell). Shown are raw data. The red line marks the mean values at each time point, demonstrating the reduction of statistical noise. Scale bars in $\mathbf{b}$ and $\mathbf{c}, 10 \mu \mathrm{m}$. important for the light-driven off-switching in Dreiklang, because it appears to be in a suitable position for a nucleophilic addition across the $\mathrm{C}=\mathrm{N}$ bond of the imidazolinone ring.

The off-state structure reveals a new water molecule ( $\mathrm{Wat}_{\mathrm{b}}$ ) displaced by $1.02 \pm 0.09 \AA$ from the position of Wat ${ }_{\mathrm{a}}$. It is held in this position by a differently configured hydrogen bonding network. We found during occupancy refinement of Wat that the occupancy was significantly smaller than 1.0 (e.g., chain C: 0.35 ), strongly indicating that this position is not always occupied by a water molecule in the off-state protein. Hence we assume that $W t_{b}$ is taken up from the environment after Wat ${ }_{a}$ has been used for the light-induced hydration of the imidazolinone ring upon switching from the on- to the off-state.

Altogether, these findings lend support to a reversible light-induced hydration/dehydration reaction of the five-membered ring. In the on-state, the chromophore exists in the protonated and the deprotonated form, resulting in absorption bands at $412 \mathrm{~nm}$ and $511 \mathrm{~nm}$, respectively. Irradiation at the 511-nm band induces fluorescence, whereas irradiation at the $412-\mathrm{nm}$ band induces a covalent modification (hydration) of the five-membered ring, resulting in a nonfluorescent chromophore absorbing at $340 \mathrm{~nm}$. Subsequent irradiation at this band results in a dehydration of the off-state chromophore converting it back into the on-state chromophore. Although the reversible water addition/elimination reaction appears to be the key factor in the unusual switching behavior of Dreiklang, it is possible that additional short-lived intramolecular rearrangements may occur, possibly including a cis-trans isomerization, structural flexibility or a strong bending of the chromophore.

\section{Use of Dreiklang for fluorescence recovery after switching}

To evaluate the properties of Dreiklang for imaging purposes, we prepared a layer of purified Dreiklang molecules and wrote complex patterns $6,30,31$ into this layer showing that Dreiklang can be exploited for reversibly recording and reading information (Fig. 3a and Supplementary Movie 1). Next, we generated several fusion proteins, namely Dreiklang-Map2, Dreiklang- $\alpha$-tubulin, Dreiklang-histone2B, keratin19-Dreiklang, vimentin-Dreiklang, $\beta$-actin-Dreiklang and Dreiklang targeted to the mitochondrial matrix (Mito-Dreiklang) and expressed these fusion proteins in cultivated mammalian cells (Fig. 3b and Supplementary Fig. 12). The fact that Dreiklang could be functionally fused to $\alpha$-tubulin and histone2B demonstrates that Dreiklang behaves as a monomer also in vivo. Targeted to the different cellular structures, Dreiklang could be switched on and off in living cells repeatedly (Fig. 3 b).

Because switching Dreiklang on and off requires UV and nearUV light, we were concerned that this irradiation might negatively affect the viability of the cells. To investigate this issue, we expressed vimentin-Dreiklang in PtK2 cells. The Dreiklang fluorescence was switched 100 times on and off in whole living cells with widefield illumination, displaying a contrast in the fluorescence signal of around 75:1 in each cycle (Supplementary Fig. 13 and Supplementary Movie 2). No major alterations in the labeled structures were observed during this period $(\sim 10 \mathrm{~min})$. Next, we targeted Dreiklang to the endoplasmic reticulum (ER) of PtK2 cells and switched the ability to fluoresce 5 or 20 times on and off (Supplementary Fig. 14). We found that five switching cycles had no detectable effect on the viability of the cells after $2 \mathrm{~h}$ compared to the control where switching was omitted. After 20 cycles, $\sim 16 \%$ of the cells were dead after $2 \mathrm{~h}$ ( $n=300$ cells, six independent experiments) and a larger and highly variable fraction of cells showed changes in their shapes. This finding demonstrates that repeated irradiation with UV light is unfavorable but does not necessarily induce cell death. However, most practical applications will require either fewer switching cycles and/or much smaller regions exposed to UV light for switching, which thus would reduce the overall light dose by at least an order of magnitude and the effects on the cellular viability accordingly.

As a demonstration that Dreiklang's properties can be exploited for repeated measurements of protein dynamics in individual living cells, we expressed Dreiklang targeted to the ER in Vero cells (Fig. 3c). Using a commercial confocal microscope, we selectively photoswitched (with $405 \mathrm{~nm}$ ) ER-Dreiklang in one region of the living cell from the fluorescent into the nonfluorescent state and followed the movement of nonswitched on-state molecules into the switched-off region by probing fluorescence with $515 \mathrm{~nm}$. After the measurement, the fluorophores in the whole cell were switched back to the initial fluorescent state with $360 \mathrm{~nm}$ using wide-field illumination; the 

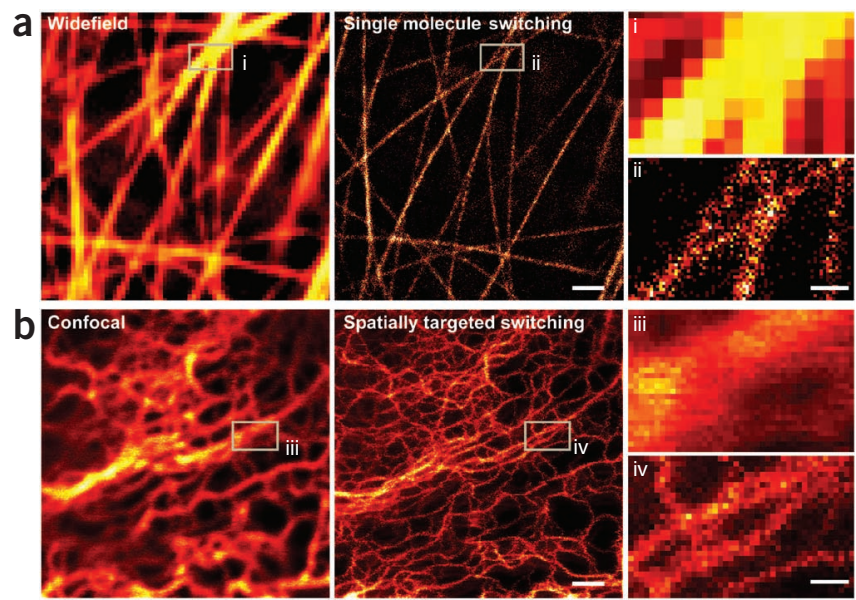

Figure 4 Super-resolution microscopy of living PtK2 cells using Dreiklang. (a) Cells expressing Dreiklang-Map2 imaged both conventionally (left) and by super-resolution microscopy based on single-molecule stochastic switching (center). (b) Keratin19-Dreiklang expressed in living cells and imaged both confocally (left) and in the RESOLFT mode (spatially targeted switching) (center). Right: magnifications of the regions indicated in the main images. Scale bars, $1 \mu \mathrm{m}$ (middle, left), $250 \mathrm{~nm}$ (right).

measurement, which was substantially shorter (65 s) than the thermal equilibration of Dreiklang to the on-state, was repeated several tens of times. We denominate this approach as fluorescence recovery after switching (FRAS). Individual measurements of cellular protein movements often exhibit statistical noise ${ }^{32-34}$, which was strongly reduced in FRAS by averaging over many switching cycles. Analogous approaches have previously been done using conventional RSFPs or photoactivatable proteins ${ }^{16,35}$.

\section{Nanoscopy with Dreiklang}

To overcome the diffraction barrier, all super-resolution (nanoscopy) concepts use a molecular mechanism to sequentially inhibit the fluorescence of adjacent features ${ }^{36}$. Two families of approaches rest on this principle. In the first family, which has been termed RESOLFT (reversible saturable optical (fluorescence) transitions between two states), the sample coordinates at which the fluorophores are on and off are predefined by a pattern of light ${ }^{6,8,36}$. In the second family encompassing (F)PALM, STORM and others ${ }^{9,10,36,37}$, individual fluorophores are switched on and off stochastically, whereby the coordinate of the emitting molecule is found using the photons detected on a camera. We reasoned that the decoupling of fluorescence switching from excitation in Dreiklang would enable additional possibilities for super-resolution microscopy.

In the stochastic methods, the number of emitted photons and the emission rate of a molecule in the on-state together determine the localization precision and the recording time. Although these parameters are difficult to control in conventional fluorescent proteins or RSFPs, in Dreiklang the on-time can be influenced by adapting the light intensities used for switching on and off, eventually even interactively in response to the local fluorophore densities. We fused Dreiklang to Map2 and expressed the fusion construct in PtK2 cells. The cells were initially irradiated for $10 \mathrm{~s}$ at $405 \mathrm{~nm}\left(0.2 \mathrm{~kW} / \mathrm{cm}^{2}\right)$ to switch the majority of Dreiklang proteins off. Subsequently we recorded 8,000 image frames (10 ms each) with irradiation at $491 \mathrm{~nm}\left(2 \mathrm{~kW} / \mathrm{cm}^{2}\right)$. Between two frames the cells were irradiated for $0.1-0.5 \mu$ s with light of $405 \mathrm{~nm}$ and/or $355 \mathrm{~nm}\left(2 \mathrm{~W} / \mathrm{cm}^{2}\right)$ to adjust the number of on-state fluorophores to less than one per diffraction area. We obtained an average localization precision of $\sim 15 \mathrm{~nm}$ using $\sim 700$ detected photons, resulting in images that were clearly superior to the conventional counterparts (Fig. 4a).

We also realized an implementation based on spatially targeted switching of the fluorophores (RESOLFT) ${ }^{6,8}$. We imaged living PtK2 cells expressing keratin19-Dreiklang by first switching Dreiklang on at $355 \mathrm{~nm}\left(1 \mathrm{~ms}, 160 \mathrm{~W} / \mathrm{cm}^{2}\right)$ using a regular shaped, diffractionlimited focus. Then, a doughnut-shaped focus of light of $405 \mathrm{~nm}$ $\left(40 \mathrm{~ms}, 110 \mathrm{~W} / \mathrm{cm}^{2}\right)$ with a central intensity minimum (zero) was used for switching Dreiklang off at the focal periphery only. Finally, fluorescence was probed by $491 \mathrm{~nm}$ excitation $\left(4 \mathrm{~kW} / \mathrm{cm}^{2}\right)$ for $10 \mathrm{~ms}$. The image was generated by scanning over the sample using this irradiation scheme at every pixel (Fig. 4b). The Dreiklang images taken in the RESOLFT mode reveal many details with a resolution of down to $\sim 35 \mathrm{~nm}$ (Supplementary Fig. 15) that are fully blurred in the corresponding confocal data. Note that the presented image data are raw.

\section{DISCUSSION}

The photoswitching of Dreiklang is based on a light-driven reversible hydration-dehydration reaction modifying the chromophore, which uniquely decouples the switching spectra from their excitation counterpart. Thus Dreiklang allows fine-tuning of the duration of the chromophore states without interference by the fluorescence excitation light.

This feature provides benefits for super-resolution microscopy techniques applying stochastic single-molecule switching (Fig. 4a) and spatially targeted switching (Fig. 4b), but should also enable applications that would be difficult or even impossible with conventional switchable fluorescent proteins. Among others, this includes FRAS (Fig. 3c), allowing multiple bleaching or photoactivation measurements in single cells, potentially enabling a new generation of single cell-based screening approaches. One may also envision sophisticated Förster resonance energy transfer (FRET) measurements with multiple fluorophores using Dreiklang as an additional switchable component. Dreiklang may also provide new avenues to multicolor applications in combination with other RSFPs or conventional fluorescent proteins. In this case, contrast may be obtained either by switching ${ }^{19}$, or by different colors or by dissimilar fluorescence lifetimes. In addition to applications in life sciences, the decoupling of switching from fluorescence excitation should also offer new options in subdiffraction reading and writing in protein layers.

Long-term protein tracking will require Dreiklang variants with longer lifetimes of the metastable states. Likewise, it is conceivable to design proteins offering even more switching cycles and operating with longer wavelengths. With its X-ray structure available, we anticipate the specific generation of further Dreiklang derivatives forming a new class of switchable fluorescent proteins.

\section{METHODS}

Methods and any associated references are available in the online version of the paper at http://www.nature.com/naturebiotechnology/.

Accession code. The atomic coordinates and structure factors have been deposited in the Protein Data Bank, http://www.pdb.org (PDB ID codes 3ST2, 3ST3 and 3ST4).

Note: Supplementary information is available on the Nature Biotechnology website.

\section{ACKNOWLEDGMENTS}

We acknowledge access to beamline BL14.2 of the BESSY II storage ring (Berlin) through the Joint Berlin MX-Laboratory sponsored by the Helmholtz Zentrum Berlin für Materialien und Energie, the Freie Universität Berlin, the HumboldtUniversität zu Berlin, the Max-Delbrück Centrum and the Leibniz-Institut für 
Molekulare Pharmakologie. We thank V. Belov for insightful discussions and F. Lavoie-Cardinal for help with the set-up for targeted switching. We acknowledge A. Schönle for providing the software ImSpector. We also thank T. Gilat, S. Löbermann, R. Pick and E. Rothermel for excellent technical assistance, H.-H. Hsiao for help in ESI-MS and H. Schill and J. Jethwa for carefully reading the manuscript. We acknowledge R.Y. Tsien for sharing the plasmid pRSET-Citrine. This work was supported by the Deutsche Forschungsgemeinschaft through the Gottfried Wilhelm Leibniz Prize (to S.W.H.) and through the DFG-Research Center for Molecular Physiology of the Brain (to S.J.).

\section{AUTHOR CONTRIBUTIONS}

G.W., M.A. and I.T. contributed equally to this work. C.E., S.W.H. and S.J. conceived the project. T.B., A.C.S., G.W., M.A., I.T., T.G., M.L. and U.P. performed all experiments. I.T. recorded the super-resolution images. Data analysis was done by T.B., A.C.S., G.W., M.A., I.T., T.G., M.L., H.U., C.E., M.C.W., S.W.H. and S.J. The manuscript was written by S.W.H. and S.J. All authors discussed the results and commented on the manuscript.

\section{COMPETING FINANCIAL INTERESTS}

The authors declare competing financial interests: details accompany the full-text HTML version of the paper at http://www.nature.com/nbt/index.html.

Published online at http://www.nature.com/nbt/index.html.

Reprints and permissions information is available online at http://www.nature.com/ reprints/index.html.

1. Tsien, R.Y. The green fluorescent protein. Annu. Rev. Biochem. 67, 509-544 (1998).

2. Lippincott-Schwartz, J., Altan-Bonnet, N. \& Patterson, G.H. Photobleaching and photoactivation: following protein dynamics in living cells. Nat. Cell Biol. S7-S14 (2003).

3. Lukyanov, K.A., Chudakov, D.M., Lukyanov, S. \& Verkhusha, V.V. Innovation: photoactivatable fluorescent proteins. Nat. Rev. Mol. Cell Biol. 6, 885-890 (2005).

4. Dickson, R.M., Cubitt, A.B., Tsien, R.Y. \& Moerner, W.E. On/off blinking and switching behaviour of single molecules of green fluorescent protein. Nature $\mathbf{3 8 8}$, 355-358 (1997)

5. Habuchi, S. et al. Reversible single-molecule photoswitching in the GFP-like fluorescent protein Dronpa. Proc. Natl. Acad. Sci. USA 102, $9511-9516$ (2005).

6. Hell, S.W., Jakobs, S. \& Kastrup, L. Imaging and writing at the nanoscale with focused visible light through saturable optical transitions. Appl. Phys. A Mater. Sci. Process. 77, 859-860 (2003).

7. Hell, S.W. Toward fluorescence nanoscopy. Nat. Biotechnol. 21, 1347-1355 (2003).

8. Hofmann, M., Eggeling, C., Jakobs, S. \& Hell, S.W. Breaking the diffraction barrier in fluorescence microscopy at low light intensities by using reversibly photoswitchable proteins. Proc. Natl. Acad. Sci. USA 102, 17565-17569 (2005).

9. Betzig, E. et al. Imaging intracellular fluorescent proteins at nanometer resolution. Science 313, 1642-1645 (2006).

10. Hess, S.T., Girirajan, T.P. \& Mason, M.D. Ultra-high resolution imaging by fluorescence photoactivation localization microscopy. Biophys. J. 91, 4258-4272 (2006).

11. Patterson, G.H. \& Lippincott-Schwartz, J. A photoactivatable GFP for selective photolabeling of proteins and cells. Science 297, 1873-1877 (2002).

12. Chudakov, D.M. et al. Photoswitchable cyan fluorescent protein for protein tracking. Nat. Biotechnol. 22, 1435-1439 (2004).
13. Subach, F.V. et al. Photoactivatable mCherry for high-resolution two-color fluorescence microscopy. Nat. Methods 6, 153-159 (2009).

14. Lukyanov, K.A. et al. Natural animal coloration can be determined by a nonfluorescent green fluorescent protein homolog. J. Biol. Chem. 275, 25879-25882 (2000).

15. Cinelli, R.A.G. et al. Green fluorescent proteins as optically controllable elements in bioelectronics. Appl. Phys. Lett. 79, 3353-3355 (2001).

16. Ando, R., Mizuno, H. \& Miyawaki, A. Regulated fast nucleocytoplasmic shuttling observed by reversible protein highlighting. Science 306, 1370-1373 (2004).

17. Henderson, J.N., Ai, H.W., Campbell, R.E. \& Remington, S.J. Structural basis for reversible photobleaching of a green fluorescent protein homologue. Proc. Natl. Acad. Sci. USA 104, 6672-6677 (2007).

18. Stiel, A.C. et al. $1.8 \AA$ bright-state structure of the reversibly switchable fluorescent protein Dronpa guides the generation of fast switching variants. Biochem. J. 402, 35-42 (2007).

19. Andresen, M. et al. Photoswitchable fluorescent proteins enable monochromatic multilabel imaging and dual color fluorescence nanoscopy. Nat. Biotechnol. 26, 1035-1040 (2008).

20. Stiel, A.C. et al. Generation of monomeric reversibly switchable red fluorescent proteins for far-field fluorescence nanoscopy. Biophys. J. 95, 2989-2997 (2008).

21. Adam, V. et al. Structural characterization of IrisFP, an optical highlighter undergoing multiple photo-induced transformations. Proc. Natl. Acad. Sci. USA 105 18343-18348 (2008).

22. Subach, F.V. et al. Red fluorescent protein with reversibly photoswitchable absorbance for photochromic FRET. Chem. Biol. 17, 745-755 (2010).

23. Sinnecker, D., Voigt, P., Hellwig, N. \& Schaefer, M. Reversible photobleaching of enhanced green fluorescent proteins. Biochemistry 44, 7085-7094 (2005).

24. Shaner, N.C. et al. Improving the photostability of bright monomeric orange and red fluorescent proteins. Nat. Methods 5, 545-551 (2008).

25. Ormö, M. et al. Crystal structure of the Aequorea victoria green fluorescent protein. Science 273, 1392-1395 (1996).

26. Griesbeck, O., Baird, G.S., Campbell, R.E., Zacharias, D.A. \& Tsien, R.Y. Reducing the environmental sensitivity of yellow fluorescent protein - Mechanism and applications. J. Biol. Chem. 276, 29188-29194 (2001).

27. Siegbahn, P.E.M., Wirstam, M. \& Zimmer, M. Theoretical study of the mechanism of peptide ring formation in green fluorescent protein. Int. J. Quantum Chem. 81 169-186 (2001).

28. Rosenow, M.A., Huffman, H.A., Phail, M.E. \& Wachter, R.M. The crystal structure of the $\mathrm{Y} 66 \mathrm{~L}$ variant of green fluorescent protein supports a cyclization-oxidationdehydration mechanism for chromophore maturation. Biochemistry 43, 4464-4472 (2004).

29. Barondeau, D.P., Kassmann, C.J., Tainer, J.A. \& Getzoff, E.D. Understanding GFP chromophore biosynthesis: controlling backbone cyclization and modifying posttranslational chemistry. Biochemistry 44, 1960-1970 (2005).

30. Irie, M., Fukaminato, T., Sasaki, T., Tamai, N. \& Kawai, T. Organic chemistry: a digital fluorescent molecular photoswitch. Nature 420, 759-760 (2002).

31. Sauer, M. Reversible molecular photoswitches: a key technology for nanoscience and fluorescence imaging. Proc. Natl. Acad. Sci. USA 102, 9433-9434 (2005).

32. Lippincott-Schwartz, J., Snapp, E. \& Kenworthy, A. Studying protein dynamics in living cells. Nat. Rev. Mol. Cell Biol. 2, 444-456 (2001).

33. Phair, R.D. \& Misteli, T. Kinetic modelling approaches to in vivo imaging. Nat. Rev Mol. Cell Biol. 2, 898-907 (2001).

34. Sprague, B.L. \& McNally, J.G. FRAP analysis of binding: proper and fitting Trends Cell Biol. 15, 84-91 (2005).

35. Chudakov, D.M., Chepurnykh, T.V., Belousov, V.V., Lukyanov, S. \& Lukyanov, K.A. Fast and precise protein tracking using repeated reversible photoactivation. Traffic 7 , 1304-1310 (2006).

36. Hell, S.W. Microscopy and its focal switch. Nat. Methods 6, 24-32 (2009).

37. Rust, M., Bates, M. \& Zhuang, X. Sub-diffraction-limit imaging by stochastic optical reconstruction microscopy (STORM). Nat. Methods 3, 793-796 (2006). 


\section{ONLINE METHODS}

Cloning. Constructs for bacterial expression. For protein expression and mutagenesis, the respective coding sequences were PCR amplified, digested and inserted into the BamHI/HindIII restriction sites of the plasmid pQE31 (Qiagen).

Constructs for expression in mammalian cells. To target Dreiklang to the lumen of the endoplasmic reticulum (ER), the coding sequence of Dreiklang was amplified by PCR using the primers 5'-CTGCAGGTCGACATGGTGAGCA AGGGCGAGGA- ${ }^{\prime}$ and $5^{\prime}$-TTCTGCGGCCGCCTTGTACAGCTCGTCCAT GCCGCCGGT-3'. The PCR fragments were digested with SalI and NotI and inserted into the vector $\mathrm{pEF} / \mathrm{myc} / \mathrm{ER}$ (Invitrogen).

To target Dreiklang to the mitochondrial matrix, the coding sequence of Dreiklang was amplified by PCR using the primers $5^{\prime}$-TCCACCG GTCGCCACCATGGTGAGCAAGGGCGAGGAGCTGTTC-3' and 5' -GTCG CGGCCGCTACTTGTACAGCTCGTCCATGCCGAGAG-3'. The PCR fragments were digested with AgeI and NotI and inserted into the vector pDsRed1Mito (Clontech), replacing the DsRed 1 sequence.

For the generation of the $\alpha$-tubulin fusion construct, the Dreiklang coding sequence was amplified by PCR using the primers 5 '-GATCCGCTAGCGCT AATGGTGAGCAAGGGCGAGGAG- ${ }^{\prime}$ and $5^{\prime}$-CACTCGAGATCTGAGTCC GGACTTGTACAGCTCGTCCATGCC- $3^{\prime}$. The PCR fragments were digested with NheI and BglII and inserted into the vector pEGFP-Tub (Clontech), replacing the EGFP sequence.

For the generation of the microtubule-associated protein 2 (Map2) fusion construct, the Map2 coding sequence (obtained from pDONR223-MAP2) was amplified by PCR using the primers $5^{\prime}$-GATCTCGAGTGATGGCAGAT

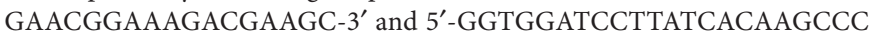
TGCTTAGCGAGTGCAGC- $3^{\prime}$. The PCR fragments were digested with XhoI and BamHI and inserted into the vector Dreiklang- $\alpha$-tubulin, replacing the $\alpha$-tubulin sequence.

For the generation of the Histon-2B fusion construct, the Histon-2B coding sequence (obtained from pDONR223-HIST1H2BN ${ }^{38}$ ) was amplified by PCR using the primers $5^{\prime}$-GATCTCGAGTGATGCCCGAGCCCTCAAA

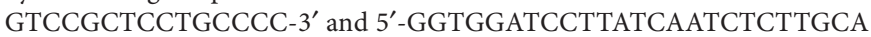
ATATAAATGTCATCTGG- $3^{\prime}$. The PCR fragments were digested with XhoI and BamHI and inserted into the vector Dreiklang- $\alpha$-tubulin, replacing the $\alpha$-tubulin sequence.

To create fusion constructs of Dreiklang with vimentin, keratin19 and beta-actin, Dreiklang was amplified using the primers $5^{\prime}$-GATCCACCGGT CGCGGCGTGAGCAAGGGCGAGGAGCTG-3' and 5' ${ }^{\prime}$-ACAACTTAAGAA CAACAATTGTTACTTGTACAGCTCGTCCATGCC-3'. The PCR fragment was cloned into the gateway destination vector pMD-tdEosFP- ${ }^{39}$ using the restriction sites AgeI and AflII, thereby replacing the tdEosFP coding sequence against the Dreiklang sequence. The final plasmids pMD-Vim-Dreiklang, pMD-Ker19-Dreiklang and PMD-ACTB-Dreiklang were constructed by gateway vector conversion (Invitrogen) using the donor vectors pDONR223-Vim, pDONR223-Krt19 and pDONR223-ACTB ${ }^{38}$.

Mutagenesis. For site-directed random mutagenesis, the QuikChange SiteDirected Mutagenesis Kit (Stratagene) was used according to the manufacturer's instructions. Error-prone random mutagenesis and directed simultaneous mutagenesis of multiple sites were performed according to standard protocols.

Protein expression and purification. Proteins were expressed in the Escherichia coli strain BL21-CodonPlus (Stratagene) and purified by Ni-NTA affinity chromatography, followed by a gel-filtration step on a Superdex 200 column (Amersham Biosciences) using a Tris-buffer (100 mM Tris- $\mathrm{HCl}$, $150 \mathrm{mM} \mathrm{NaCl}, \mathrm{pH} 7.5)$.

Protein characterization. Semi-native PAGE. Proteins (10 $\mu \mathrm{g}$ each) were loaded onto $15 \%$ polyacrylamide gels containing $0.1 \%$ sodiumdodecyl sulfate (SDS). For loading, proteins were taken up in a $60 \%$ (wt/vol) sucrose solution. As size standards, purified monomeric EGFP and Citrine, dimeric dTomato and tetrameric DsRed were used.
Protein and chromophore maturation. An overnight TOP10 E. coli culture (Invitrogen) transformed with the pBad-Dreiklang plasmid, grown at $37^{\circ} \mathrm{C}$ in LB medium supplemented with ampicillin was used to inoculate $200 \mathrm{ml}$ of $\mathrm{LB}_{\text {Amp }}$ medium. After the bacterial cells were grown at $37^{\circ} \mathrm{C}$ to an $\mathrm{OD}_{600}$ of 0.5 , protein expression was induced by adding arabinose to a final concentration of $0.2 \%$. After $4 \mathrm{~h}$ of further incubation at $37^{\circ} \mathrm{C}$, cells were pelleted and disrupted. Dreiklang proteins were immediately purified using His SpinTrap columns (GE Healthcare) within $20 \mathrm{~min}$ and kept strictly at $4{ }^{\circ} \mathrm{C}$. The purified protein solution was diluted in buffer (final buffer concentration: $20 \mathrm{mM}$ sodium phosphate, $500 \mathrm{mM} \mathrm{NaCl}, 30 \mathrm{mM}$ imidazole $\mathrm{pH}$ 7.5) and then filled into QS cuvettes, which were incubated at $30^{\circ} \mathrm{C}$ and $37^{\circ} \mathrm{C}$, respectively. For assessment of maturation, fluorescence emission spectra were taken with a Varian Cary Eclipse fluorescence spectrometer (Varian), at the indicated time points.

Absorption and emission spectra. Absorption and emission spectra were recorded on a Varian Cary 4000 UV/VIS spectrophotometer and a Varian Cary Eclipse fluorescence spectrometer (Varian), respectively. Quantum yields and extinction coefficients were determined in comparison to EGFP ${ }^{40}$. The indicated values are the means of three independent protein purifications.

Photoswitching. Switching of Dreiklang or Citrine fluorescence was recorded either on E. coli colonies grown on LB-agar plates (Fig. 1c,d and Supplementary Fig. 4) or in various buffered protein solutions. For recording of the switching at various temperatures purified Dreiklang was taken up in $100 \mathrm{mM}$ Tris$\mathrm{HCl}, 150 \mathrm{mM} \mathrm{NaCl}, \mathrm{pH}$ 7.5. A modified computer-controlled fluorescence microscope (Leica Microsystems) equipped with a $50 \times$ NA 0.5 or a $20 \times$ NA 0.4 air objective lens and three $100 \mathrm{~W}$ Hg lamps was used for data acquisition. Different excitation filters $(365 / 25,405 / 10,595 / 10$, LOT Oriel) and shutters (Melles Griot) in front of each lamp facilitated the controlled on- and offswitching and the fluorescence recording. Fluorescence was recorded using a photomultiplier tube (HR9306-0, Hamamatsu) behind a $525 \mathrm{~nm}$ longpass detection filter (HQ 525 LP, AHF Analysentechnik).

Relaxation half-times. For the determination of the relaxation half-time from the nonfluorescent off-state into the fluorescent on-state (thermal equilibrium), purified Dreiklang (at pH 7.5) was switched completely into the off-state and the relaxation to the equilibrium state was followed by consecutive short pulses or continuous irradiation with light of $515 \mathrm{~nm}$. For details on the used intensities and the timing see Supplementary Table 1. These experiments were performed at the indicated temperatures in a temperature-controlled environment.

The thermal relaxation of Dreiklang from the light-driven off-state into the equilibrium state in living cells was determined on PtK2 cells expressing Dreiklang in the ER using a wide-field epifluorescence microscope. For the measurements, Dreiklang was first switched into the nonfluorescent state $(4 \mathrm{~s}, 405 / 10 \mathrm{~nm}$, $\left.80 \mathrm{~W} / \mathrm{cm}^{2}\right)$. Subsequently, images were recorded $\left(515 / 10 \mathrm{~nm}, 2.6 \mathrm{~W} / \mathrm{cm}^{2}\right)$ at different time points to monitor the thermal relaxation. Finally, the cells were irradiated with light of $365 / 25 \mathrm{~nm}\left(5 \mathrm{~s}, 3.4 \mathrm{~W} / \mathrm{cm}^{2}\right)$ and an image providing the maximal fluorescence was recorded. For analysis, regions of interest were selected within the cells. For background subtraction the fluorescence signal next to the respective cells was used.

Imaging. For imaging of mammalian cells, Vero cells (kidney epithel cells of Cercopithecus aethiops) or PtK2 cells (kidney epithel cells of Potorous tridactylis) were transfected with the respective plasmids by the Nanofectin Kit (PAA) according to the manufacturer's instructions. The cells were propagated in DMEM medium with GlutaMAX and $4.5 \mathrm{~g} / \mathrm{l}$ glucose, $10 \%$ (vol/vol) FCS, $1 \mathrm{mM}$ sodium pyruvate, $50 \mathrm{mg} / \mathrm{ml}$ penicillin and $50 \mathrm{mg} / \mathrm{ml}$ streptomycin. Cells were grown on coverslips in Petri dishes at $37^{\circ} \mathrm{C}(310 \mathrm{~K})$ under $90 \%$ humidity and $5 \% \mathrm{CO}_{2}$.

Repeated switching of Dreiklang embedded in polyacrylamide (PAA). A solution of Dreiklang molecules was embedded in PAA (final concentration: 15\% PAA in $1.5 \mathrm{M}$ Tris/ $\mathrm{HCl}$ buffer, $\mathrm{pH} 8.8$ ) and squeezed under a coverslip to ensure a layer thickness of $\sim 1 \mu \mathrm{m}$. For reading and writing a Leica SP5 beam scanning confocal microscope equipped with a $40 \times$ NA 1.25 oil immersion objective 
was used. The following settings were used: pinhole, $1.48 \mathrm{AU}$; pixel size, $378.8 \mathrm{~nm} \times 378.8 \mathrm{~nm}$; scan speed, $400 \mathrm{~Hz}$. Fluorescence was detected between 517 and $575 \mathrm{~nm}$. Except for smoothing and contrast stretching, no further image processing was applied.

To generate the PacMan-movie (Fig. 3a and Supplementary Movie 1), each individual image was written using a group of ROIs, defining the positions where Dreiklang was transferred from the on- to the off-state by irradiation with $405 \mathrm{~nm}$ laser-light $\left(4.3 \mathrm{MW} / \mathrm{cm}^{2}\right)$. Subsequently the written image was recorded with $515 \mathrm{~nm}$ laser-irradiation $\left(2.4 \mathrm{~kW} / \mathrm{cm}^{2}\right)$ only. In the next step all proteins were switched back to the fluorescent state with $360 \mathrm{~nm}$ widefield illumination $\left(0.1 \mathrm{~W} / \mathrm{cm}^{2}, \mathrm{BP} 360 \mathrm{~nm} / 40 \mathrm{~nm}, 5 \mathrm{~s}\right)$ and the next image was recorded.

Widefield measurements. For widefield imaging (Fig. 3b) a Leica DM6000 epifluorescence microscope (Leica) equipped with a $100 \times$ NA 1.40 oil immersion objective lens and a DFC350 FX camera (Leica) was used. Cells were irradiated for $300 \mathrm{~ms}$ with near-UV light $\left(420 / 30 \mathrm{~nm}, 15 \mathrm{~W} / \mathrm{cm}^{2}\right)$ to switch the Dreiklang molecules to the nonfluorescent state and $300 \mathrm{~ms}$ with UV light $\left(360 / 40 \mathrm{~nm}, 5 \mathrm{~W} / \mathrm{cm}^{2}\right)$ to switch the proteins back to the fluorescent state. The fluorescence images were recorded with green light $\left(495 / 15 \mathrm{~nm}, 13 \mathrm{~W} / \mathrm{cm}^{2}\right)$. For fluorescence detection, a $510 \mathrm{~nm}$ dichroic mirror and a 530/30 nm detection filter were used. The length of the image acquisition time was adjusted to the brightness of every sample and was generally between 50 and $150 \mathrm{~ms}$.

To record 100 switching cycles of Dreiklang in living mammalian cells (Supplementary Fig. 13 and Supplementary Movie 2), the same microscope settings were used. Switching times were $800 \mathrm{~ms}$ (near-UV) and $100 \mathrm{~ms}$ (UV). Images were recorded with an acquisition time of $100 \mathrm{~ms}$.

Confocal microscopy. Confocal imaging (Supplementary Fig. 12) was performed with a beam scanning confocal microscope (SP5, Leica Microsystems) equipped with a UV-corrected NA 1.4 oil immersion lens ( $63 \times$ HCX PL APO). All imaging was performed at $\sim 25^{\circ} \mathrm{C}$. For excitation, light of $515 \mathrm{~nm}$ was used, fluorescence was detected between 520 and $600 \mathrm{~nm}$.

FRAS. Imaging was performed with a beam scanning confocal microscope (SP5, Leica Microsystems) equipped with a UV-corrected NA 1.4 oil immersion lens $\left(63 \times\right.$ HCX PL APO). All imaging was performed at $\sim 25^{\circ} \mathrm{C}$. The following microscope settings were used: pinhole, 1 Airy unit (AU); pixel size, $160.8 \mathrm{~nm} \times 160.8 \mathrm{~nm}$; scan speed, 1,400 Hz. Fluorescence was detected between 519 and $590 \mathrm{~nm}$.

For each measurement, the Dreiklang molecules in an ROI $(1.3 \mu \mathrm{m} \times$ $10 \mu \mathrm{m}$ ) were switched to the nonfluorescent state using $405 \mathrm{~nm}$ laser light $\left(2.7 \mathrm{MW} / \mathrm{cm}^{2}\right)$. The fluorescence was recorded by excitation with $515 \mathrm{~nm}$ laser light $\left(1.5 \mathrm{~kW} / \mathrm{cm}^{2}\right)$. Finally, the Dreiklang molecules in the whole sample were switched to the fluorescent state with $360 \mathrm{~nm}($ BP360 nm/40 nm; $1 \mathrm{~s}$; $0.6 \mathrm{~W} / \mathrm{cm}^{2}$ ) widefield illumination. This imaging sequence was repeated several times on the same cell. In every sequence a second ROI on the other side of the same cell and a third ROI in a neighboring cell were analyzed. No further data processing was performed, except background subtraction and normalization to 1 .

Analysis of the influence of irradiation on cell viability. Imaging was performed with an epi-fluorescence microscope equipped with a custom-built incubator chamber to maintain the temperature at $37^{\circ} \mathrm{C}$ and a water immersion objective (HCX APO L 20×/0.50 W U-V-I). At the end of the experiment, cells were incubated for 15 min with Sytox AADvanced Dead Cell Stain (Invitrogen). Switching was carried out by irradiation with light of $405 / 10 \mathrm{~nm}\left(24 \mathrm{~W} / \mathrm{cm}^{2}\right.$ for $4 \mathrm{~s})$ to switch off and $365 / 25 \mathrm{~nm}\left(1 \mathrm{~W} / \mathrm{cm}^{2}\right.$ for $\left.6 \mathrm{~s}\right)$ to switch on. Images were recorded with light of $515 / 10 \mathrm{~nm}\left(1.3 \mathrm{~W} / \mathrm{cm}^{2}\right)$ using an Alta CCD-camera (Apogee Instruments). For the statistical analysis, only cells expressing Dreiklang were considered.

Single-molecule switching nanoscopy. Single-molecule-switching imaging was performed on a home-built setup (Supplementary Fig. 16) that has been detailed before ${ }^{39}$. The nanoscope was equipped with continuous-wave lasers emitting at $488 \mathrm{~nm}$ (Ar-Kr laser Innova 70C-5, Coherent) for fluorescence excitation, at $355 \mathrm{~nm}$ (solid state, Zouk, Cobolt) for switching on and at $405 \mathrm{~nm}$ (laser diode, Oxxius; Laser2000) for switching off. Furthermore, it was equipped with an oil immersion objective lens (HCX PL APO 100×/1.4 oil, Leica) for creating an approximately $(12 \times 12) \mu \mathrm{m}^{2}$ large excitation spot and with a fast EMCCD camera (IXON-DU-897-CSO-BV, Andor Technology) for detection of fluorescence in epi-direction. The use of an EMCCD camera at high gain ensured that the read-out noise was negligible. The frame rate of the camera was optimized to minimize the background $(100 \mathrm{~Hz})$. The fluorescence was further cleaned by inserting a band pass filter (532/211; AHF Analysentechnik) to minimize the out-of-band background. Image acquisition, localization, spectral assignment, image reconstruction and simulations of two-dimensional histograms were performed as described before ${ }^{41}$. Image acquisition was started after switching off the protein ensemble in a preselected cell region until clear signatures of single emitters were observed. Switching of the lasers was controlled by a fast shutter (Uniblitz, Acal BFI Optilas) for the $491 \mathrm{~nm}$ laser, an acousto-optical modulator (AOM, AAA; Pegasus Optik) for the $355 \mathrm{~nm}$ laser and direct modulation of the $405 \mathrm{~nm}$ diode laser. Synchronization of the camera and the laser switching was realized by the IMSPECTOR software.

We defined the average number of photons $n_{f l}=720$ detected per single emitter by the expectation value of the geometrical distribution of photon counts per spots detected over the whole image-recording process. For the respective wide-field images, we simply added up the photons detected for all single-molecule events.

RESOLFT imaging. We modified a home-built confocal microscope (Supplementary Fig. 17). The microscope provided diffraction-limited foci of $355 \mathrm{~nm}$ (solid state, Zouk, Cobolt, for switching Dreiklang into the fluorescent state) and $491 \mathrm{~nm}$ laser light (solid state, Calypso, Cobolt, for probing the fluorescence of Dreiklang). A third laser path provided a doughnut-shaped focus at $405 \mathrm{~nm}$ (solid state, CrystaLaser) with a central intensity minimum for switching Dreiklang off at the focal periphery only. The $405 \mathrm{~nm}$ beam passed through a $2 \pi$ phase ramp (407 nm mask, vortex plate VPP- $1 \mathrm{~b}, \mathrm{RPC}$ Photonics) for creating a doughnut in the focal plane. A quarter and a half wave plate (B. Halle) were introduced in front of the objective for circularizing the polarization of all beams. Illumination powers and times were controlled by an acousto-optical-tunable filter (AOTF, AAA, Pegasus Optik) for the $491 \mathrm{~nm}$ and by acousto-optical modulators (AOM, AAA, Pegasus Optik) for the 405 and $355 \mathrm{~nm}$ light. Scanning of the probe was realized by a three-axis piezo table (NanoMax 311/M, Thorlabs). The experiment was synchronized by the IMSPECTOR software.

Mass spectrometry. For the determination of the molecular mass of the Dreiklang protein in its equilibrium, switched-off and switched-on states, $160 \mu \mathrm{g}$ of the protein in $10 \mathrm{mM}$ ammonium acetate buffer were diluted with or without prior photoswitching in $18 \%$ (vol/vol) acetonitrile/10 mM ammonium acetate. The photoswitching was done as described above.

ESI-MS analysis was performed under standard conditions on an LTQOrbitrap XL instrument (ThermoFisherScientific) in high-resolution mode $(100,000)$ and on a Q-ToF ultima (Waters company) with infusion pump and static needle, respectively. Spectra were recorded and deconvoluted by using the manufacture's software tools (Excalibur and MaxEnd, respectively).

For the MALDI-ToF/ToF analysis, equilibrium-state Dreiklang was digested with trypsin (enzyme to substrate ratio, 1:20) at $37^{\circ} \mathrm{C}$ overnight. Tryptic peptides were separated by nano liquid chromatography (LC), mixed with matrix, spotted onto MALDI target and analyzed by MALDI MS on a 4800 Proteome Analyser (ABSciex), as described previously ${ }^{42}$. MSMS spectra were annotated manually.

Crystallographic analysis. After purification, a Dreiklang protein solution was concentrated to $\sim 35 \mu \mathrm{g} / \mu \mathrm{l}$ by ultrafiltration and taken up in $20 \mathrm{mM}$ Tris/ $\mathrm{HCl}, 120 \mathrm{mM} \mathrm{NaCl}, \mathrm{pH}$ 7.5. The protein was crystallized by sitting drop vapor diffusion at $20^{\circ} \mathrm{C}(293 \mathrm{~K})$ with a reservoir solution containing $15 \%(\mathrm{wt} / \mathrm{vol})$ PEG 3350, $0.2 \mathrm{M} \mathrm{KH}_{2} \mathrm{PO}_{4}$ and 3\% (wt/vol) D-(+)-trehalose-dihydrate. The crystallization solution was cryoprotected by adding propane-1,3-diol to a final concentration of $25 \%$ (wt $/ \mathrm{vol}$ ).

For the structure determination of Dreiklang in the on- and the off-states, a single Dreiklang protein crystal was manually transferred into a vessel with 
cryoprotected crystallization solution and then switched to the nonfluorescent state by irradiation with blue light $\left(405 \pm 10 \mathrm{~nm}, 110 \mathrm{~W} / \mathrm{cm}^{2}\right)$ until the fluorescence signal reached a minimum. After switching the crystal, it was transferred within $10 \mathrm{~s}$ into liquid nitrogen, flash frozen, and diffraction data were collected at $100 \mathrm{~K}$. Following the data collection of the off-state structure, the same crystal was unmounted, transferred back into cryoprotected crystallization solution kept at $25^{\circ} \mathrm{C}$ and immediately switched to the fluorescent-state by irradiation with UV light $\left(365 \pm 25 \mathrm{~nm}, 1.4 \mathrm{~W} / \mathrm{cm}^{2}\right)$ until the fluorescence signal reached a maximum. Subsequently the crystal was again flash frozen in liquid nitrogen and another diffraction data set was collected.

The equilibrium structure of Dreiklang was determined on crystals that have not been irradiated with light.

All diffraction data sets were collected on beamline 14-2 at BESSY (Berliner Elektronenspeicherring-Gesellschaft für Synchrotronstrahlung) using a MAR $225 \mathrm{~mm}$ CCD detector. Details for the refinement and crystallographic data are given in Supplementary Table 2. The data were processed using the XDS program package ${ }^{43}$. The crystal structures were solved by molecular replacement with PHASER ${ }^{44}$ using the structure coordinates of Citrine (PDB ID 1 HUY; ${ }^{26}$ ) as a model omitting the chromophore and the water molecules. The sequence was manually adjusted and fitted to the $2 F_{\mathrm{o}}-F_{\mathrm{c}}$ and $F_{\mathrm{o}}-F_{\mathrm{c}}$ electron densities with $\mathrm{COOT}^{45}$. Atomic B-factors and coordinates were refined automatically with PHENIX.REFINE ${ }^{46}$. TLS (translation-librationscrew) refinement cycles were performed. Refinement was complemented by manual model building with COOT. Waters were built automatically with PHENIX.REFINE and completed manually. During the entire refinement procedure, $5 \%$ of randomly selected reflections were set aside for monitoring of the $R_{\text {free }}$ factor.
Finally, the omitted chromophore was placed manually into vacant patches of the $2 F_{\mathrm{o}}-F_{\mathrm{c}}$ and $F_{\mathrm{o}}-F_{\mathrm{c}}$ electron densities. The chromophore restraints for the on-state fluorophore were generated with PRODRG ${ }^{47}$, and the restraints of the off-state chromophore were generated with PHENIX.ELBOW ${ }^{48}$.

38. Lamesch, P. et al. hORFeome v3.1: a resource of human open reading frames representing over 10,000 human genes. Genomics 89, 307-315 (2007).

39. Testa, I. et al. Nanoscale separation of molecular species based on their rotational mobility. Opt. Express 16, 21093-21104 (2008)

40. Patterson, G.H., Knobel, S.M., Sharif, W.D., Kain, S.R. \& Piston, D.W. Use of the green fluorescent protein and its mutants in quantitative fluorescence microscopy. Biophys. J. 73, 2782-2790 (1997).

41. Bossi, M. et al. Multicolor far-field fluorescence nanoscopy through isolated detection of distinct molecular species. Nano Lett. 8, 2463-2468 (2008).

42. Richter, F.M., Sander, B., Golas, M.M., Stark, H. \& Urlaub, H. Merging molecular electron microscopy and mass spectrometry by carbon film-assisted endoproteinase digestion. Mol. Cell. Proteomics 9, 1729-1741 (2010).

43. Kabsch, W. Automatic processing of rotation diffraction data from crystals of initially unknown symmetry and cell constants. J. Appl. Cryst. 26, 795-800 (1993).

44. McCoy, A.J. et al. Phaser crystallographic software. J. Appl. Cryst. 40, 658-674 (2007).

45. Emsley, P. \& Cowtan, K. Coot: model-building tools for molecular graphics. Acta Crystallogr. D Biol. Crystallogr. 60, 2126-2132 (2004).

46. Adams, P.D. etal. PHENIX: a comprehensive Python-based system for macromolecular structure solution. Acta Crystallogr. D Biol. Crystallogr. 66, 213-221 (2010).

47. Schuttelkopf, A.W. \& van Aalten, D.M. PRODRG: a tool for high-throughput crystallography of protein-ligand complexes. Acta Crystallogr. D Biol. Crystallogr. 60, 1355-1363 (2004).

48. Moriarty, N.W., Grosse-Kunstleve, R.W. \& Adams, P.D. electronic ligand builder and optimization workbench (eLBOW): a tool for ligand coordinate and restraint generation. Acta Crystallogr. D Biol. Crystallogr. 65, 1074-1080 (2009). 